\title{
Exploring and managing psychiatric symptoms in ICU healthcare professionals during the ongoing COVID-19 pandemic: A focused review and guideline
}

\author{
Ashish Sarangi MD, Dalynn Kim MS
}

\begin{abstract}
Introduction: The COVID-19 pandemic has ravaged the healthcare system and stretched resources thin. The impact on healthcare staff working in the intensive care unit setting has been especially devastating.

Objective: To conduct a review of the impact of the pandemic on healthcare personnel in the intensive care unit setting in an effort to collect information to inform various stakeholders.

Design: Focused literature and abstract review

Conclusion: This article highlights the association between critical care as it relates to trauma and COVID-19 and point stakeholders toward opportunities for anticipating and managing secondary effects in an effort to promote psychological adaptation.
\end{abstract}

Keywords: COVID-19 pandemic; ICU, depression, anxiety, burnout, healthcare workers

\section{BACKGROUND}

Healthcare professionals (HCPs) are the first responders during viral outbreaks, putting their health at risk and adapting to novel situations as they emerge to care for patients. It comes as no surprise to learn that HCPs experience high levels of negative psychiatric symptoms related to depression, anxiety, posttraumatic stress, and much more during global and epidemic infectious outbreaks. ${ }^{1-3}$ Among HCPs, those employed in intensive care units (ICUs) are disproportionately affected by mental health morbidities compared to HCPs working in non-acute settings. ${ }^{2,4-7}$ Medical employees in high-risk settings are also at increased risk for experiencing more severe symptoms of depression, anxiety, PTSD, and burnout than their low-risk setting colleagues. ${ }^{5}$

Corresponding author: Ashish Sarangi

Contact Information: Ashish.Sarangi@ttuhsc.edu DOI: 10.12746/swrccc.v9i41.853
The 2020 COVID-19 pandemic presents its own particular set of challenges for HCPs, particularly those employed in ICUs and equivalent critical care settings. A seemingly perpetual shortage of ICU beds, personal protective equipment (PPE), and mechanical ventilators, along with a relatively high hospitalization rate of COVID-19 patients has placed an enormous strain on hospitals worldwide. ${ }^{8}$ Healthcare professionals in ICUs have been particularly burdened by this viral outbreak due to the frontline nature of their work and the rapid changes that were implemented in ICUs to care for the surge of seriously ill patients. Because ICU stressors such as bed allocations, patient death, and long work hours have been associated with high levels of emotional exhaustion, disengagement, and an increased risk for depression, it is crucial to survey the potential mental health outcomes of ICU HCPs to effectively implement appropriate protective workplace measures. ${ }^{9}$ This focused review analyzes the current literature on the prevalence and quality of psychiatric symptoms, such as depression, fear and anxiety, stress and moral suffering, burnout, and sleep disturbances in healthcare professionals employed in ICUs and critical care wards during the 2020 COVID-19 pandemic. 


\section{Methods}

Information was gathered through a literature search conducted on PubMed from 12 Feb 2020 to 14 Feb 2021. Key search words included: COVID-19 AND ICU AND mental health OR psychology. The following criteria were used to filter for relevant research: full-text accessible, peer reviewed primary research published in English, and conducted between March and December 2020 in the context of COVID-19 and psychological factors. Publications were further filtered for specific cohorts/sub-cohorts that met the following criteria: Healthcare professionals employed in adult intensive care units (ICUs) treating COVID-19 patients. It is important to recognize some data reported in this review was sourced from studies that categorized ICU and employees with employees of other high-exposure "frontline" departments associated with the treatment of COVID-19 (e.g., Infectious Disease Wards, Emergency Departments [EDs], and COVID-19 Isolation Wards). Inclusion of data from these studies was considered justifiable due to their close working relationships with ICUs during the pandemic.

\section{RESULTS}

A total of 230 studies were reviewed to yield 28 eligible studies, including five exceptions whose cohorts included staff from other high-exposure hospital departments, such as Respiratory Ward, Emergency Department (ED), Infectious Disease Ward, Isolation Ward, and High Acuity Unit (HAU). A summary of the literature search is depicted by the PRISMA Flow Diagram (Diagram one). Occupational titles included

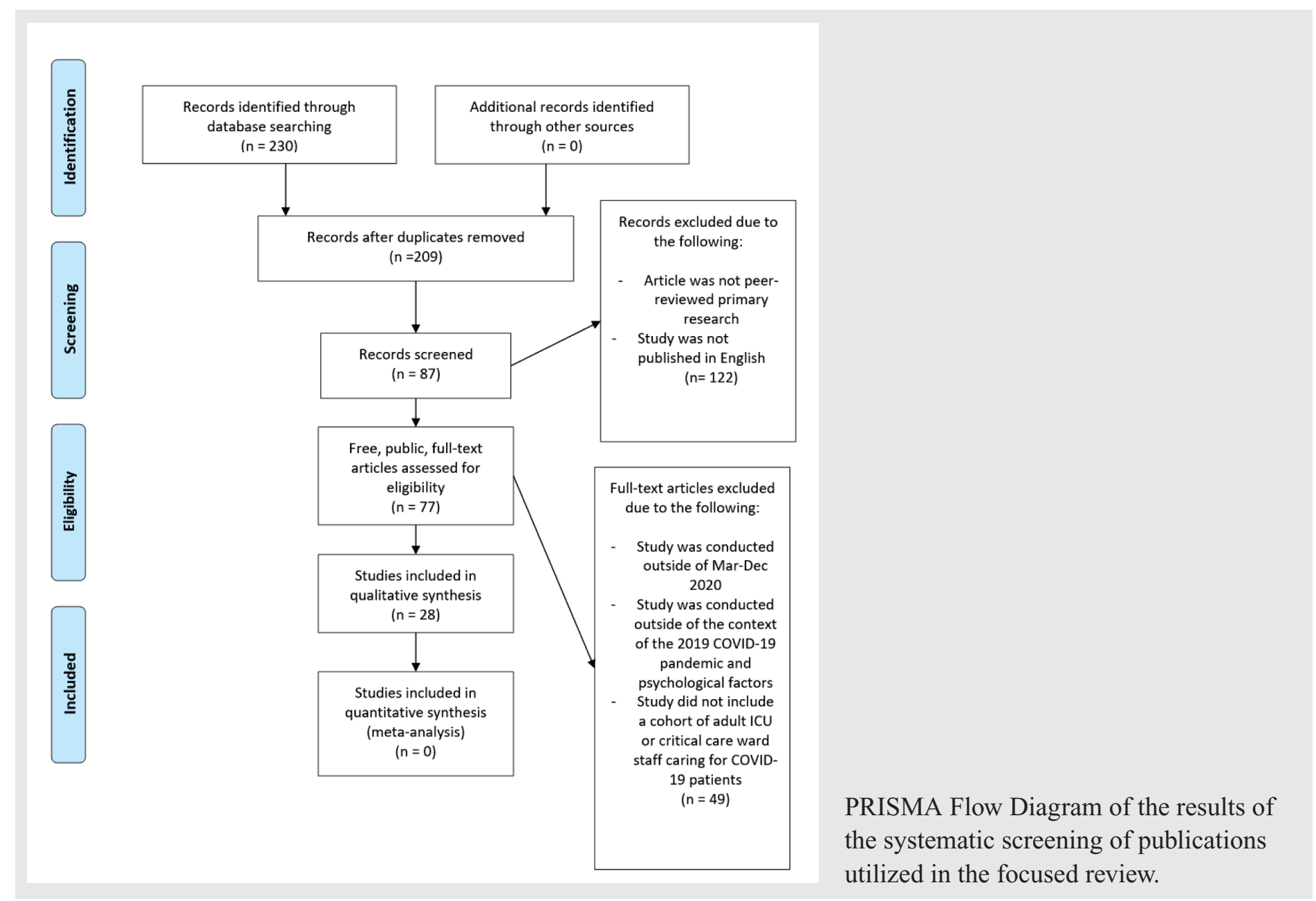


but were not limited to physicians, nurses, nursing assistants, residents, physician assistants, and nurse practitioners, and medical technicians. Three studies focused solely on physicians, seven solely on nursing staff, and three solely on residents. Cohorts from at least 15 unique countries were included in the review.

\section{Discussion}

\section{DEPRESSION}

The 2020 COVID-19 pandemic affected ICUs worldwide on a scale that few were prepared for, and its effect on the mental health of ICU HCPs is available in the medical literature. Based on existing data, an estimated $24 \%$ to $57 \%$ of ICU medical staff have experienced at least mild symptoms of depression during the pandemic. ${ }^{10-15}$ One UK study reported $26 \%$ of doctors felt moderately depressed despite reporting higher levels of mental well-being compared to their collegues. ${ }^{13}$ Alarmingly, studies revealed $2 \%$ to $13 \%$ of ICU HCPs experienced thoughts of self-harm or thoughts of better off being dead, with nurses reporting a prevalence as high as $19 \% .{ }^{13,16}$

Prevalence or severity in depressive symptoms was significantly associated with ICU employment, female identification, employment as a nurse, and presence of other psychiatric comorbidities. ${ }^{10,13,15}$ Similarly, HCPs employed in high-risk wards such as the ICU, ED and Respiratory Department were significantly more likely to suffer from depression compared to non-clinical staff, and one Polish study discovered more than $99 \%$ of frontline employees met the Patient Health Questionnaire (PHQ-9) criteria for depressive symptoms. ${ }^{1,7}$

Prevalence of related depressive symptoms in ICU HCPs was demonstrated in one Chinese study stating $59 \%$ of nurses reported decreased appetite, $55 \%$ felt fatigued, and $26 \%$ admitted to frequent crying, while a Turkish study reported $30 \%$ of HCPs felt hopelessness and despair. ${ }^{12,16}$ In addition, two substantial European studies measured the prevalence of substance use among ICU HCPs during the pandemic. One study claimed more than one in three intensivists took sleeping pills, $10 \%$ smoked cigarettes, and $12 \%$ reported excessive alcohol intake two months into the pandemic. ${ }^{11}$ Another nationwide French study reported alcohol and illicit drugs consumption jumped from $5.2 \%$ to $23.7 \%$ after the pandemic began. ${ }^{10}$ Protective factors against depressive symptoms included higher levels of mental well-being and male identification. ${ }^{10,13}$

\section{AnXIETY AND FEAR}

The reported prevalence of anxiety symptoms in ICU HCPs varied from $20 \%$ to $78 \% .^{10-15,17}$ The literature suggests anxiety was associated with ICU employment, employment as a nurse, female identification, and presence of other psychiatric comorbidities. ${ }^{10,11,13,14,17}$ In contrast, one small Italian study found no significance between ICU employment and severity of anxiety, while another study evaluating 596 Turkish ICU nurses reported a negative correlation between ICU employment and level of anxiety. ${ }^{12,18}$ Along with ICUs, employment in high-risk wards were significantly associated with a greater prevalence and severity of anxiety, and these workers were twice as likely to experience anxiety and 1.4 times more likely to feel fear than non-clinical employees. ${ }^{1,7}$ However, a Saudi Arabian study found no significant difference in the intensity of anxiety, fear, or isolation between physicians working in COVID-19 ICUs and isolation wards and those employed in lowerrisk wards. ${ }^{19}$

Unsurprisingly, a major source of fear and anxiety for HCPs stemmed from the possibility of infection by SARS-CoV-2 and subsequently spreading the virus to coworkers and family members. ${ }^{10,20-23}$ In the U.S. $26 \%$ of ICU HCPs felt concerned about their hospital's ability to keep their employees safe. ${ }^{23}$ Another major concern for HCPs during the pandemic was adequacy of ICU training; one French study stated HCPs who felt they received inadequate ICU training were twice as likely to experience anxiety symptoms. The same study reported one in three HCPs felt they were not adequately trained to work in the ICU, with $43 \%$ having no prior ICU experience before the pandemic and $48 \%$ reporting less than a year of experience at the time of the study. ${ }^{24}$

Healthcare professionals in ICUs experiencing fear and anxiety also suffered from psycho-somatic symptoms including shortness of breath, muscle tension, 
and changes in appetite or gastrointestinal trouble. ${ }^{16,25}$ Similarly, $38 \%$ of medical staff reported experiencing at least one nervous breakdown. ${ }^{25}$ Although few studies explored the role of protective factors against anxiety in ICU HCPs, increased mental well-being and male identification was significantly associated with lower levels of anxiety. ${ }^{10.13}$

\section{STRESS AND MORAL SUFFERING}

The reported prevalence of mental stress in ICU HCPs was between $22 \%$ and $73 \% .{ }^{12,14,20}$ A Chinese study revealed one in five highly skilled and resilient ICU nurses still experienced harmful levels of stress as measured by the Perceived Stress Scale (CPSS-14). ${ }^{20}$ Significant factors associated with an increased risk for emotional distress included female identification, high patient volume, and insufficient feedback from supervisors. ${ }^{23}$ In addition, one small Romanian study reported ICU HCPs affected by misinformation of COVID-19 in the media were significantly more likely to experience higher stress levels. ${ }^{26}$ Other reported sources of stress during the pandemic included lack of resources such as PPE and ICU beds. ${ }^{23,27}$

Clinical stress was also analyzed using the criteria for post-traumatic stress (PTS). The literature reported a prevalence of PTS symptoms between $27 \%$ and $74 \%$ in ICU HCPs. ${ }^{13,14,24,28}$ Furthermore, $49 \%$ of nurses and $32 \%$ of doctors met the Post-Traumatic Stress Disorder checklist (PCL-6) criteria for probable post-traumatic stress disorder (PTSD), and while the majority of ICU nurses reported they were willing to care for COVID-19 patients, they were significantly less willing than their Internal Medicine colleagues. ${ }^{13,29}$ The prevalence or severity of PTS symptoms was significantly related to ICU employment, employment as a nurse, other psychiatric comorbidities, and not feeling adequately trained. ${ }^{13,24}$

Moral suffering/distress was another parameter of stress highlighted in some studies. One Netherlands teaching hospital revealed the prevalence of moral distress was significantly associated with administering "suboptimal care" to patients due to resource shortage, postponing inevitable death, and working with under-qualified $\mathrm{HCPs}^{30}$ Similarly, $58 \%$ HCPs experienced moral suffering due to the decisions of other medical workers in the ICU. ${ }^{31}$ Psychological stress and moral suffering may have been exacerbated by staff reassignment to the ICU during the pandemic, with an average increase of $37 \%$ in trainee reassignment to ICUs in especially burdened U.S. hospitals. ${ }^{32}$ Consequently, one Saudi Arabian study revealed over $80 \%$ of reassigned surgical residents reported making difficult decisions about resource allocation among sick patients, and felt their inexperience in the ICU put their patients and colleagues in possible harm's way. ${ }^{33}$

\section{BURNOUT}

Studies suggested prevalence of burnout symptoms in ICU HCPs ranged from $36 \%$ to $58 \%$. ${ }^{11,23,30}$ As outlined by Maslach Burnout Inventory (MBI) General use or Human Services workers version, $37 \%$ to $57 \%$ of HCPs from various frontline wards felt emotionally exhausted, $25 \%$ felt depersonalized towards the needs of others, and $40 \%$ felt cynicism about their line of work. ${ }^{25,34}$ Employment in an ICU and employment as a nurse positively correlated with prevalence or severity of burnout. ${ }^{11,18,23}$ Despite a higher prevalence of burnout in nurses, one Dutch cross sectional, including 233 participants, suggested ICU physicians were 3.5 times at higher risk of experiencing burnout relative to nurses. ${ }^{30}$ Observation of the effects on hospital reassignment on mental health revealed over half of surgical trainees reassigned to a London ICU believed the relocation negatively impacted their psychological state, citing burnout as a major cause, despite an almost unanimous agreement that the clinical experience would benefit them as future surgeons. ${ }^{21}$

The pandemic also forced ICU workers to compromise on personal priorities, possibly contributing to the high prevalence of burnout reported in the literature. One Spanish study reported less than 3\% of ICU HCPs were regularly afforded time to unwind between shifts. ${ }^{31}$ Comparatively, $23 \%$ of HCPs reported having virtually no time to rest and $22 \%$ reported an inability to care for their families. ${ }^{10}$ Personal gratification was a significant protective factor against burnout, while identifying with the female gender was significantly associated with increased severity of emotional exhaustion. ${ }^{25}$ 


\section{SLEEP DISTURBANCES}

Because poor sleep quality can often exacerbate psychiatric morbidities and vice versa, the pandemic's effect on sleep in ICU HCPs becomes important. Current data suggested the prevalence of sleep disturbances ranged from $27 \%$ to $68 \%$. $^{10,12,16,17,25,31}$ One Polish study encompassing $441 \mathrm{HCPs}$ from various frontline wards reported $100 \%$ of HCPs met the Insomnia Severity Index (ISI-7) criteria for symptoms of sleep disturbance. ${ }^{7}$ The prevalence and severity of sleep disturbance among HCPs was significantly associated with ICU employment. ${ }^{17,22}$ Furthermore, ICU HCPs negatively impacted by misinformation in the media experienced a significantly higher prevalence of insomnia compared to workers not impacted by false news. ${ }^{26}$ High-risk ward employment, including the ICU, was also significantly associated with an increased prevalence or severity in sleep disturbances compared to low-risk wards, and Isolation Ward HCPs were 1.7 times more likely to experience insomnia compared to other high-risk wards. ${ }^{7,35}$

\section{RECOMMENDATIONS FOR MANAGEMENT OF MENTAL HEALTH OUTCOMES FOR HCPS IN ICUS}

Based on the current review of mental health outcomes of ICU HCPS during the pandemic, one of the most consistent findings among studies was the association between higher frequency or severity of psychiatric symptoms and ICU employment status. Therefore, it may be beneficial to establish mental health intervention at the level of the workplace. Offering employees referral-based or internal counseling services would provide a direct route toward psychiatric intervention or psychiatric evaluation, and previous studies have demonstrated that direct professional assistance for common psychiatric disorders (e.g., anxiety and PTSD) can improve workplace efficiency and outcomes in HCPs. ${ }^{36}$ Understandably, this option may not be widely feasible due to hospital resource constraints; instead, a suitable alternative such as organized therapeutic writing workshops may offer some solution. Writing therapy has been successfully implemented in patients with depression and anxiety, it is less resource-intense, and sessions can be modulated to accommodate busy hospital schedules ${ }^{37}$ Another relatively convenient and cost-effective method of mental health intervention is the use of web or app-based therapies that employees can access through hospital-funded subscriptions. Online therapy offers employees a discrete and flexible tool that can be used as a stand-alone or adjunct resource to self-evaluate their mental health status and seek assistance for a broad range of topics from mindfulness meditation to suicide prevention. ${ }^{38}$ In addition, it is recommended workplaces do not neglect the data on increased substance use in ICU HCPs during the pandemic and provide appropriate assistance and education to employees. Hospitals can direct employees to self-referral state programs for clinicians struggling with substance use disorders or offer employee insurance plans that cover substance use treatment options. ${ }^{38,39}$ Finally, it is suggested ICU departments not only offer access to mental health care, but additionally facilitate preventative measures in the workplace that enhance mental and physical well-being and increase morale. Promotion of healthy and supportive workplace practices can act as protective factors against modifiable mental health risks; practical implementations include encouraging debriefing sessions, offering HCPs opportunities to spend more time with their families (e.g., "mental health" sick days), creating quiet, comforting retreats in the hospital, and providing employees healthy food options and access to physical fitness facilities. ${ }^{27,39}$

\section{Conclusion}

The psychological burdens experienced by ICU HCPs during the 2020 COVID-19 pandemic have exposed areas of vulnerability that warrant special consideration when implementing mental health services for frontline workers and designing future studies. Current research suggests that females and nurses are disproportionately affected by symptoms of depression, anxiety, stress, and burnout, establishing a vital need to identify the unique risk factors of being a woman or nurse ICU HCP during the pandemic. ${ }^{10,11,13,23,25,30}$ The results of the literature search also emphasized the impact of perceived skill level and ICU training on mental health, suggesting the need to develop effective training modules for newly 
reassigned ICU HCPs to maximize their confidence and ensure optimal patient care and safety. $24,30,33$ Another consideration highlighted by the pandemic was the influence of protective factor against psychiatric morbidities and general need for further exploration of protective factors, such as positive psychological traits or lifestyle modifiers. Last, the literature has revealed a serious concern for suicide risk in ICU HCPs during these extraordinary times. More research is needed to assess the unique risk factors of suicide facing healthcare workers employed in high-risk settings. ${ }^{13,16}$ To provide ICU HCPs optimal psychiatric support, we must continue to utilize current scientific information to tailor mental health accommodations to their specific needs.

Article citation: Sarangi A, Kim D. Exploring and managing psychiatric symptoms in ICU healthcare professionals during the ongoing COVID-19 pandemic: A focused review and guideline. The Southwest Respiratory and Critical Care Chronicles 2021;9(41):28-34

From: Menninger Department of Psychiatry \& Behavioral Sciences (AS), Baylor College of Medicine, Houston, TX; Texas Tech University Health Sciences Center (DK), Lubbock, Texas

Submitted: $4 / 25 / 2021$

Accepted: 9/28/2021

Reviewer: Cheryl Erwin JD, PhD

Conflicts of interest: none

This work is licensed under a Creative Commons

Attribution-ShareAlike 4.0 International License.

\section{REFERENCES}

1. Lu W, Wang H, Lin Y, et al. Psychological status of medical workforce during the COVID-19 pandemic: A crosssectional study. Psychiatry Res 2020;288:112936.

2. Philip J, Cherian V. Factors affecting the psychological well-being of health care workers during an epidemic: a thematic review. Indian J Psychol Med 2020;42(4):323-333.

3. Stuijfzand S, Deforges C, Sandoz V, et al. Psychological impact of an epidemic/pandemic on the mental health of healthcare professionals: a rapid review. BMC Public Health 2020;20(1):1230.

4. Mealer ML, Shelton A, Berg B, et al. Increased prevalence of post-traumatic stress disorder symptoms in critical care nurses. Am J Respir Crit Care Med 2007;175(7):693-697.
5. Serrano-Ripoll MJ, Meneses-Echavez JF, Ricci-Cabello I, et al. Impact of viral epidemic outbreaks on mental health of healthcare workers: a rapid systematic review and metaanalysis. Journal of Affective Disorders 2020; 277:347-357.

6. Vandevala T, Pavey L, Chelidoni O, et al. Psychological rumination and recovery from work in intensive care professionals: associations with stress, burnout, depression and health. J intensive care 2017;5(16).

7. Wa kowicz P, Szyli ska A, Rotter I. Assessment of mental health factors among health professionals depending on their contact with COVID-19 Patients. Int J Environ Res Public Health 2020;17(16):5849.

8. Willan J, King AJ, Jeffery K, et al. Challenges for NHS hospitals during COVID-19 epidemic. BMJ 2020;368:m1117.

9. Coomber S, Todd C, Park G, et al. Stress in UK intensive care unit doctors. British Journal of Anaesthesia 2002;89(6): 873-881.

10. Azoulay E, Cariou A, Bruneel F, et al. Symptoms of anxiety, depression, and peritraumatic dissociation in critical care clinicians managing patients with COVID-19. A cross-sectional study. Am J Respir Crit Care Med. 2020;202(10):13881398.

11. Azoulay E, De Waele J, Ferrer R, et al. Symptoms of burnout in intensive care unit specialists facing the COVID-19 outbreak. Ann. Intensive Care 2020;10(10). doi: 10.1186/s13613020-00722-3.

12. Bahadir-Yilmaz E, Yüksel A. State anxiety levels of nurses providing care to patients with COVID-19 in Turkey. Perspect Psychiatr Care. 2020. doi: 10.1111/ppc.12661.

13. Greenberg N, Weston $D$, Hall C, et al. Mental health of staff working in intensive care during COVID-19. Occup Med (Lond) 2021.

14. Crowe S, Howard AF, Vanderspank-Wright B, et al. The effect of COVID-19 pandemic on the mental health of Canadian critical care nurses providing patient care during the early phase pandemic: A mixed method study. Intensive Crit Care Nurs 2021;63:102999.

15. Ma Y, Rosenheck R, He H. Psychological stress among health care professionals during the 2019 novel coronavirus disease outbreak: Cases from online consulting customers. Intensive Crit Care Nurs 2020;61:102905.

16. Zhu Y, Chen T, Wang J, et al. How critical activities within COVID-19 intensive care units increase nurses' daily occupational calling. J Appl Psychol 2021;106(1):4-14.

17. Jain A, Singariya G, Kamal M, et al. COVID-19 pandemic: Psychological impact on anaesthesiologists. Indian J Anaesth 2020;64(9):774-783.

18. Buselli R, Corsi M, Baldanzi S, et al. Professional Quality of Life and Mental Health Outcomes among Health Care Workers Exposed to Sars-Cov-2 (Covid-19). Int J Environ Res Public Health 2020;17(17):6180. 
19. Al Sulais E, Mosli M, AlAmeel T. The psychological impact of COVID-19 pandemic on physicians in Saudi Arabia: A cross-sectional study. Saudi J Gastroenterol 2020;26(5): 249-255.

20. Leng M, Wei L, Shi X, et al. Mental distress and influencing factors in nurses caring for patients with COVID-19. Nurs Crit Care 2020. doi: 10.1111/nicc.12528.

21. Payne A, Rahman R, Bullingham R, et al. Redeployment of surgical trainees to intensive care during the COVID-19 pandemic: evaluation of the impact on training and wellbeing. J Surg Educ 2020. doi: 10.1016/j.jsurg.2020.09.009.

22. Saracoglu KT, Simsek T, Kahraman S, et al. The psychological impact of COVID-19 disease is more severe on intensive care unit healthcare providers: a cross-sectional study. Clin Psychopharmacol Neurosci 2020;18(4):607-615.

23. Sharma M, Creutzfeldt CJ, Lewis A, et al. Healthcare professionals' perceptions of critical care resource availability and factors associated with mental well-being during COVID19: Results from a US survey. Clin Infect Dis 2020. doi: $10.1093 / \mathrm{cid} / \mathrm{ciaa} 1311$.

24. Caillet A, Coste C, Sanchez R, et al. Psychological impact of COVID-19 on ICU caregivers. Anaesth Crit Care Pain Med 2020 Dec;39(6):717-722.

25. Barello S, Palamenghi L, Graffigna G. Burnout and somatic symptoms among frontline healthcare professionals at the peak of the Italian COVID-19 pandemic. Psychiatry Res 2020;290:113-129.

26. Secosan I, Virga D, Crainiceanu ZP, et al. Infodemia: another enemy for Romanian frontline healthcare workers to fight during the COVID-19 outbreak. Medicina (Kaunas) 2020;56(12):679.

27. Ffrench-O'Carroll R, Feeley T, Tan MH, et al. Psychological impact of COVID-19 on staff working in paediatric and adult critical care. Br J Anaesth 2021;126(1):e39-e41.

28. Pasin L, Sella N, Correale C, et al. Pandemic COVID-19: the residents' resilience. Acta Biomed 2020;91(4):e2020120.

29. Wu, B, Zho, Y, Xu, D, et al. Factors associated with nurses' willingness to participate in care of patients with COVID 19: A survey in China. J Nurs Manag 2020; 28:1704-1712.
30. Kok N, van Gurp J, Teerenstra S, et al. Coronavirus Disease 2019 immediately increases burnout symptoms in icu professionals: a longitudinal cohort study. Crit Care Med 2021. doi: 10.1097/CCM.0000000000004865.

31. González-Gil MT, González-Blázquez C, Parro-Moreno AI, et al. Nurses' perceptions and demands regarding COVID-19 care delivery in critical care units and hospital emergency services. Intensive Crit Care Nurs 2021;62:102966.

32. Ellison EC, Spanknebel K, Stain SC, et al. Impact of the COVID-19 pandemic on surgical training and learner wellbeing: report of a survey of general surgery and other surgical specialty educators. J Am Coll Surg 2020;231(6):613-626.

33. Aljehani YM, Othman SA, Telmesani NK, et al. Stress and psychological resilience among general surgery residents during COVID-19 pandemic. Saudi Med J 2020;41(12): 1344-13449.

34. Lasalvia A, Amaddeo F, Porru S, et al. Levels of burn-out among healthcare workers during the COVID-19 pandemic and their associated factors: a cross-sectional study in a tertiary hospital of a highly burdened area of north-east Italy. BMJ Open 2021;11(1):e045127.

35. Zhang C, Yang L, Liu S, et al. Survey of insomnia and related social psychological factors among medical staff involved in the 2019 novel Coronavirus disease outbreak. Front Psychiatry 2020;11(306). doi: 10.3389/fpsyt.2020.00306.

36. Wagner SL, Koehn C, White MI, et al. Mental health interventions in the workplace and work outcomes: a bestevidence synthesis of systematic reviews. Int J Occup Environ Med 2016;7(1):1-14.

37. Mugerwa S, Holden JD. Writing therapy: a new tool for general practice?. Br J Gen Pract 2012;62(605):661-663.

38. Bansal $\mathrm{P}$, Bingemann TA, Greenhawt $\mathrm{M}$, et al. Clinician wellness during the COVID-19 pandemic: extraordinary times and unusual challenges for the allergist/immunologist. J Allergy Clin Immunol Pract 2020;8(6):1781-1790.

39. Goetzel RZ, Roemer EC, Holingue $C$, et al. Mental health in the workplace: a call to action proceedings from the Mental Health in the Workplace-Public Health Summit. J Occup Environ Med 2018;60(4):322-330. 